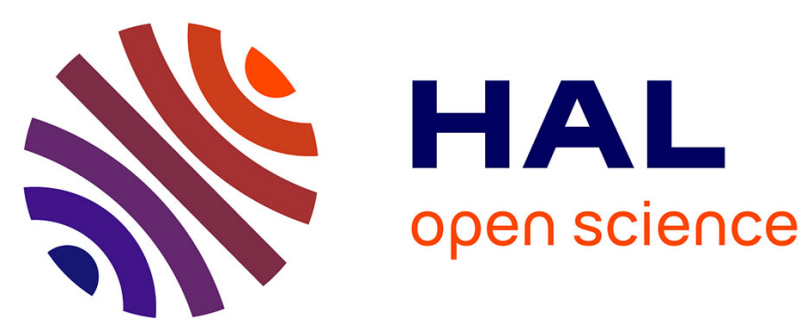

\title{
La détermination du nombre des délégués au sein des structures intercommunales: une application de l'indice de pouvoir de Banzhaf
}

Franck Bisson, Jean Bonnet, Dominique Lepelley

\section{- To cite this version:}

Franck Bisson, Jean Bonnet, Dominique Lepelley. La détermination du nombre des délégués au sein des structures intercommunales: une application de l'indice de pouvoir de Banzhaf. Revue d'économie régionale et urbaine, 2004, 2, pp.259-282. hal-00149378

\section{HAL Id: hal-00149378 \\ https://hal.science/hal-00149378}

Submitted on 12 Nov 2007

HAL is a multi-disciplinary open access archive for the deposit and dissemination of scientific research documents, whether they are published or not. The documents may come from teaching and research institutions in France or abroad, or from public or private research centers.
L'archive ouverte pluridisciplinaire HAL, est destinée au dépôt et à la diffusion de documents scientifiques de niveau recherche, publiés ou non, émanant des établissements d'enseignement et de recherche français ou étrangers, des laboratoires publics ou privés. 


\title{
La détermination du nombre des délégués au sein des structures
} intercommunales : une application de l'indice de pouvoir de Banzhaf

\section{The determination of the number of delegates inside the inter-communities structures : an application of the Banzhaf power index}

\author{
par \\ Franck Bisson \\ Doctorant \\ BDI CNRS \\ Jean Bonnet \\ Maître de Conférences \\ GEMMA, (CNRS UMR 6154) \\ Dominique Lepelley \\ Professeur \\ GEMMA, (CNRS UMR 6154) \\ Université de Caen \\ Esplanade de la paix \\ 14032, Caen Cedex, France
}

Publié dans la Revue d'Economie Régionale et Urbaine, n², pp. 259-282, 2004.

E-mails :

bisson@econ.unicaen.fr

bonnet@econ.unicaen.fr

lepelley@econ.unicaen.fr

Mots clés : Indices de pouvoir, représentativité, intercommunalité, application.

$\underline{\text { Key words }}$ : Power indices, representation, inter-communities, application.

Classification JEL : D7 


\section{1) Introduction}

Le développement important du nombre des structures intercommunales en France, a été favorisé par les incitations financières résultant de la loi Chevènement. De 269 groupements de communes à fiscalité propre en 1992, on est passé à 2175 au 1er janvier 2002, représentant plus de $73 \%$ de la population française. Cependant, lors d'enquêtes récentes, (Enquête du Forum pour la gestion des villes, 1997, Consultation nationale de l'Assemblée des Communautés de France ADCF- en 2001) les responsables des collectivités locales (élus et directeurs généraux) mettent en avant la perte de pouvoir pour l'élu municipal et la peur de la ville centre pour expliquer que certaines réticences se font encore jour. Il est vrai que se rassembler au sein d'une structure intercommunale se traduit obligatoirement par une dilution du pouvoir de décision de chaque commune. Le problème de la représentativité au sein de l'intercommunalité est donc un problème crucial qui peut se traduire par l'échec de la mise en place d'une structure voire, le plus souvent peut-être, par l'échec de la fusion de deux structures déjà existantes ou de l'extension du périmètre d'une communauté. Dans les faits, la représentativité des différentes communes au sein de la structure intercommunale est essentiellement laissée à la libre appréciation des exécutifs locaux qui, d'une manière générale, tiennent compte de la population pour fixer le nombre de délégués par commune ${ }^{1}$. La théorie des jeux coopératifs fournit un outil -les indices de pouvoirsusceptible de mesurer le degré de contrôle de chaque commune sur les décisions prises collectivement. Cette approche nous a permis dans un article récemment publié (BONNET, LEPELLEY, 2001) d'étudier la représentativité de chacune des communes au sein des établissements publics de coopération intercommunale (EPCI) de Basse-Normandie. Dans ce texte nous nous proposons de prolonger cette analyse préliminaire dans deux directions : en premier lieu, afin de prendre en compte les limites de notre démarche (en particulier l'absence d'associations préférentielles entre certaines communes), nous présentons une étude sur les coalitions au sein de la communauté d'agglomération du Grand Caen qui nous permet de mieux rendre compte des enjeux de pouvoir qui peuvent se nouer dans la réalité. En second lieu nous tentons de répondre à la question suivante : comment définir la meilleure distribution du nombre des délégués au sein des structures intercommunales? Il s'agit de proposer une ou plusieurs distributions des délégués au sein du conseil communautaire qui permette d'atteindre la structure de pouvoir choisie. Les élus locaux peuvent ainsi choisir une cible (par exemple le poids en terme de population de chaque commune) et en déduire, grâce à la minimisation d'un indicateur d'écart à la cible, une ou plusieurs distributions optimales du nombre des délégués.

Dans un premier point, nous rappelons la méthode de calcul des indices de pouvoir et l'appliquons aux EPCI de Basse-Normandie et en particulier celle du Grand Caen en prenant en compte les coalitions les plus plausibles (section 2).

Dans un deuxième point, nous présentons la problématique permettant de déterminer la ou les distribution(s) optimale(s) des délégués au sein des structures intercommunales (section 3) et nous l'appliquons au cas d'une communauté de communes et d'une communauté d'agglomération bas-normandes (section 4). 


\section{2) La mesure du pouvoir dans les systèmes de décision collective : une application aux EPCI de Basse-Normandie}

\section{1) L'indice de Banzhaf}

La première partie de notre étude présente l'indice de BANZHAF qui permet de mesurer le pouvoir a priori de chacune des communes au sein de l'établissement public de coopération intercommunale. La théorie des jeux coopératifs permet de modéliser très simplement les processus de décision collective dans une assemblée ou dans un comité, et plus généralement, dans toute structure usant du vote pour prendre une décision. Typiquement, les modèles utilisés supposent que les décisions sont prises selon le schéma suivant : une proposition est faite à l'assemblée ; chaque commune (ou joueur) vote " oui " ou " non " (mais ne s'abstient jamais) et la proposition est acceptée ou refusée en fonction des règles qui prévalent. Ce sont ces règles, qui précisent la composition et/ou la taille des coalitions permettant à une proposition d'être acceptée, que nous désignons quand nous parlons de système de décision collective. Nous nous intéresserons dans ce qui suit à une classe particulière de systèmes de décision à savoir les systèmes de vote pondéré (ou jeux de vote pondéré). Dans ce type de système, les communes ont des pondérations différentes dans la décision collective ; une proposition est alors acceptée si la somme des poids associés aux communes qui lui sont favorables est au moins égale à un certain seuil. Un tel système est entièrement défini par une liste $\left[q ; w_{1}, w_{2}, \ldots, w_{m}\right]$ dans laquelle $q$ désigne le quota exigé pour qu'une décision l'emporte, et $w_{l}, \ldots, w_{m}$ sont les poids des $m$ communes. L'ensemble constitué de toutes les communes est noté $N$ et tout sous-ensemble $S$ de $N$ de communes qui votent dans le même sens constitue une coalition. Une coalition est dite gagnante si la somme des poids des communes qui la constituent est supérieure ou égale au quota ; dans le cas contraire, elle est dite perdante. Comment mesurer le pouvoir des votants dans un tel système de décision collective ? Si chaque commune dispose d'un vote $\left(w_{i}=l \quad \forall i\right)$ et si la règle majoritaire est utilisée, il est clair que chacune a le même " montant" de pouvoir. L'intuition pourrait suggérer que si la commune $i$ a trois fois plus de poids que la commune $j$, alors son pouvoir est trois fois plus grand que celui de $\mathrm{j}$. L'examen du jeu $[3 ; 1,1,3]$ suffit à prouver le caractère erroné de cette intuition : dans ce jeu, la troisième commune (celle dont le poids est égal à 3) détient la totalité du pouvoir ; bien que disposant d'un poids non nul, les communes 1 et 2 ont un pouvoir nul en ce sens qu'elles n'ont aucun contrôle sur la décision qui sera prise. Les poids des communes ne donnent donc pas une évaluation correcte de leur pouvoir, de leur influence dans le processus de prise de décision. Afin de mesurer le pouvoir (entendu au sens suggéré dans l'exemple qui précède), les spécialistes de théorie des jeux ont proposé divers " indices ". Le plus connu est dû à BANZHAF (1965). Pour illustrer le raisonnement de cet auteur, nous considérons l'exemple d'un jeu à trois communes défini comme suit : $[51 ; 50,49,1]$. Selon BANZHAF, le pouvoir d'une commune est proportionnel au nombre de coalitions dans lesquelles elle est critique. Etant donnée une coalition gagnante, une commune est dite critique pour cette coalition si sa défection rend la coalition perdante. Dans notre exemple, il y a trois coalitions gagnantes (c'est à dire trois coalitions qui permettent d'obtenir le quota, soit 51 voix) : $C 1=\{J 1, J 2, J 3\}, C 2=\{J 1, J 2\}, C 3=\{J 1, J 3\}$.

(en gras les communes critiques dans chacune des coalitions gagnantes)

La commune $J 1$ est critique dans chacune de ces coalitions ; $J 2$ n'est critique que dans la coalition $C 2$ et $J 3$ n'est critique que dans la coalition $C 3$. L'indice normalisé de BANZHAF, noté $\beta$ (i), $i$ en référence à la commune, est obtenu en rapportant le nombre de fois où la commune $i$ est critique au nombre de fois où une commune quelconque s'avère être critique (ici en l'occurrence 5 fois en tout). 
Dans l'exemple, nous obtenons :

$\beta(1)=3 / 5$,

$\beta(2)=\beta(3)=1 / 5$.

L'indice de BANZHAF donne donc dans cet exemple les résultats suivants : la commune $J 1$ dispose de l'essentiel du pouvoir de vote (60\% selon BANZHAF) et les communes $J 2$ et $J 3$, bien qu'ayant des poids fort différents, ont en réalité le même pouvoir de vote, soit $20 \%$ chacune. Cet indice a été largement utilisé pour étudier la répartition du pouvoir au sein de diverses instances internationales, en particulier au sein du Conseil de l'Union Européenne (voir par exemple WIDGREN 1994, ou LARUELLE et WIDGREN, 1998). Nous nous proposons, dans ce qui suit, d'appliquer l'indice de BANZHAF à l'analyse du pouvoir des différentes communes au sein des différentes communautés de Basse-Normandie et en particulier dans la communauté d'agglomération du Grand Caen. Nous supposerons que les délégués d'une même commune votent «en bloc » sur chaque proposition : le poids $w_{i}$ de la commune $i$ sera considéré comme égal au nombre de délégués de cette commune. Cette hypothèse est forte et limite sans doute la portée de notre étude. Nous la croyons cependant relativement naturelle, du moins en première analyse, et les témoignages que nous avons pu recueillir dans diverses E.P.C.I. tendent à la conforter.

\section{2) Application aux EPCI de Basse-Normandie}

La Basse-Normandie se caractérise par un nombre de communes important. Selon le recensement de 1990, elle se classait à la neuvième position régionale en terme de nombre de communes alors qu'elle ne se classait qu'en quinzième position en terme de superficie et en dixseptième position en terme de population. Le classement est resté identique selon le recensement général de la population de 1999. Aussi la région comporte des communes de faible taille démographique (en moyenne 784 habitants en 1999 alors que la moyenne française était de 1600 habitants à la même date). L'intercommunalité est alors apparue comme un moyen de répondre à l'émiettement communal et la Basse-Normandie compte à l'heure actuelle 117 structures intercommunales (fin septembre 2002) de développement plutôt récent (MOULIN, 2002).

Nous présentons dans un premier paragraphe les principaux résultats obtenus dans BONNET et LEPELLEY (2001). Ces résultats donnent une première illustration de l'intérêt que peut avoir l'usage de l'indice de Banzhaf dans l'analyse du pouvoir au sein des structures intercommunales. Dans un second paragraphe nous prolongeons cette analyse en considérant que toutes les coalitions ne sont pas également probables au sein d'une intercommunalité particulière. Le cas de la communauté d'agglomération du Grand Caen nous permet d'illustrer les conséquences de la prise en compte d'associations préférentielles.

\subsubsection{Calcul des indices de pouvoir et résultats généraux}

Notre étude porte sur la situation de l'intercommunalité en Basse-Normandie en 1999, soient 16 E.P.C.I. dans le département du Calvados, 39 dans le département de l'Orne et 47 dans le département de la Manche. Dans un premier temps, nous nous sommes intéressés à la procédure de vote à la majorité simple (50\% des voix plus une) qui constitue la règle de prise de décisions au sein de la structure intercommunale lorsque celle-ci est constituée. Pour chaque E.P.C.I., nous avons calculé les indices de BANZHAF. Nous avons ensuite effectué un test du Khi2 entre la distribution des indices de pouvoir normalisés et la répartition en pourcentage du nombre des délégués au sein de l'E.P.C.I. censée représenter la distribution attendue du pouvoir. 
Le même test a été effectué entre la distribution du pouvoir mesuré par les indices et la répartition des communes en pourcentage de la population.

Tableau 1

Exemple de calcul d'indices de pouvoir pour la communauté de communes du Cingal (Calvados)

\begin{tabular}{|c|c|c|c|c|c|}
\hline Communes & Population & Délégués & Population(\%) & Délégués (\%) & Banzhaf \\
\hline Bretteville-sur-Laize & 1348 & 4 & $33,5 \%$ & $25,0 \%$ & $28,3 \%$ \\
\hline Gouvix & 879 & 3 & $21,8 \%$ & $18,8 \%$ & $18,3 \%$ \\
\hline Saint-Sylvain & 871 & 3 & $21,6 \%$ & $18,8 \%$ & $18,3 \%$ \\
\hline Barbery & 500 & 2 & $12,4 \%$ & $12,5 \%$ & $11,7 \%$ \\
\hline Moulines & 217 & 2 & $5,4 \%$ & $12,5 \%$ & $11,7 \%$ \\
\hline Fresney-le-Vieux & 210 & 2 & $5,2 \%$ & $12,5 \%$ & $11,7 \%$ \\
\hline Totaux & 4025 & 16 & $100 \%$ & $100 \%$ & $\begin{array}{c}\text { Majorité } \\
\text { simple:9 }\end{array}$ \\
\hline Test du Khi2 & \multicolumn{7}{|l|}{$\begin{array}{l}\text { Variable test : délégués en \%. Les indices calculés représentent-ils une } \\
\text { distribution similaire au poids des délégués au sein de la C.D.C.? }\end{array}$} & Oui \\
& $\begin{array}{l}\text { Variable test : population en \%. Les indices calculés représentent-ils } \\
\text { une distribution similaire au poids de la population en pourcentage ? }\end{array}$ & Non \\
\hline
\end{tabular}

Dans l'exemple présenté au tableau 1, la communauté de communes du Cingal est constituée de 6 communes. La commune la plus importante en terme de population (Brettevillesur-Laize) se voit attribuer le plus grand nombre de délégués, 4, deux communes moyennes se voient attribuer 3 délégués et les trois petites communes, 2 délégués. Selon l'indice de BANZHAF la plus grande commune obtient $28,3 \%$ du pouvoir de vote pour $25 \%$ du total des délégués et $33,5 \%$ du total de la population. Les deux communes moyennes obtiennent 18,3\% du pouvoir de vote pour $18,8 \%$ du poids en délégués et environ $21,7 \%$ du poids de la population totale. Enfin les trois plus petites communes se voient attribuer $11,7 \%$ du pouvoir de vote pour un poids de $12,5 \%$ du total des délégués et de $12,4 \%$ de la population pour la plus grande des trois. Les résultats du test de similarité entre la distribution des indices de pouvoir et la répartition en pourcentage du nombre total des délégués, qui représente une distribution attendue du pouvoir, nous indiquent dans ce cas particulier qu'il n'y pas de différence significative au seuil de 5\%. Par contre il existe une différence significative si la variable test est la répartition de la population (en particulier, cette différence est due à la sureprésentation des deux plus petites communes -avec seulement $5,4 \%$ et $5,2 \%$ de la population elles obtiennent $11,7 \%$ de pouvoir de vote-). Les calculs ont été effectués pour l'ensemble des E.P.C.I. de la Basse-Normandie. Le tableau 2 nous donne les résultats du test du Khi2.

Tableau 2

Pourcentage d'E.P.C.I. pour lesquels il n'existe pas de distorsion significative de pouvoir par le calcul des indices de pouvoir

\begin{tabular}{|c|c|c|}
\hline \multicolumn{3}{|c|}{ Test du Khi2 } \\
\hline Variable test & Délégués en \%age & Population en \%age. \\
\hline Calvados & $80,0 \%$ & $40,0 \%$ \\
\hline
\end{tabular}




\begin{tabular}{|c|c|c|}
\hline Orne & $89,7 \%$ & $10,3 \%$ \\
\hline Manche & $91,5 \%$ & $29,8 \%$ \\
\hline Total & $91,1 \%$ & $23,8 \%$ \\
\hline
\end{tabular}

Dans environ $90 \%$ des cas, les indices de pouvoir retracent bien la distribution en pourcentage du nombre des délégués au sein de l'E.P.C.I. Par contre les indices de pouvoir représentent imparfaitement la distribution de la population en pourcentage -dans environ 3 cas sur 4, le test du Khi2 nous amène à rejeter l'hypothèse d'égalité des distributions-. Cette conclusion est somme toute logique puisque les indices de pouvoir sont calculés sur la base des poids donnés par le nombre de délégués de chaque commune ; or ces nombres ne traduisent le plus souvent que très imparfaitement l'importance de chaque commune en terme de population. Ces premiers résultats nous amènent à considérer qu'un grand nombre de configurations sont des configurations pour lesquelles les indices de pouvoir des communes au sein de l'E.P.C.I. rendent bien compte de la distribution en pourcentage du nombre des délégués.

Les E.P.C.I. pour lesquels la distribution du pouvoir n'est pas identique à la distribution des délégués peuvent être classés en 2 catégories, ceux pour lesquels la commune centre a un poids en nombre de délégués supérieur à $35 \%$ et une autre catégorie qui regroupe des cas particuliers dans lesquels les distorsions sont liées soit au faible nombre de communes, soit à la très petite taille de certaines d'entre elles.

Dans le premier cas, il y a une sur-représentation de la commune-centre en terme de pouvoir. Le tableau 3 regroupe les cas relevant de cette catégorie.

Tableau 3

Indices de pouvoir de la commune-centre lorsque celle-ci est représentée par plus de $35 \%$ des délégués au sein de l'E.P.C.I.

\begin{tabular}{|c|c|c|c|c|}
\hline Commune-centre & Population & Population \% & Délégués \% & Banzhaf \\
\hline Saint-Lô & 20090 & $72,78 \%$ & $48,28 \%$ & $90,00 \%$ \\
\hline Argentan & 16596 & $82,95 \%$ & $47,50 \%$ & $97,30 \%$ \\
\hline Vire & 13869 & $73,74 \%$ & $45,45 \%$ & $83,80 \%$ \\
\hline Flers & 16947 & $59,49 \%$ & $45,45 \%$ & $95,20 \%$ \\
\hline Saint-Sauveur-le-Vicomte & 2204 & $61,46 \%$ & $44,44 \%$ & $82,70 \%$ \\
\hline Caen & 103545 & $57,5 \%$ & $37,1 \%$ & $81,4 \%$ \\
\hline
\end{tabular}

On peut constater ici que lorsque la commune centre a un nombre de délégués important (plus de 35\% du total des délégués), elle obtient en général un pouvoir de vote beaucoup plus important que son poids en délégués. En effet il lui suffit d'attirer très peu de communes pour obtenir la majorité.

\subsection{2) Les coalitions au sein de la communauté d'agglomération du Grand Caen :}

Une des limites aux calculs précédents est de considérer que les différentes communes n'ont pas de préférences dans leurs associations. Or il apparaît que des coalitions d'intérêts multiples peuvent se créer que ce soient des coalitions d'ordre politique, géographique, fiscal, économique, voire historique. Ces coalitions ne sont pas permanentes mais elles entraînent un changement dans les rapports de force ainsi qu'il est possible de l'illustrer dans le cas de la communauté d'agglomération du Grand-Caen ${ }^{2}$. 
Tableau 4

\section{Le pouvoir de vote au sein de la Communauté d'Agglomération du Grand Caen en l'absence de coalitions}

\begin{tabular}{|c|c|c|c|c|}
\hline Communes & \% population & Nbr délégués & \% délégués & Banzhaf (BAN) \\
\hline Caen (CAE) & 57,5 & 26 & 37,1 & 81,4 \\
\hline Hérouville (HER) & 12,1 & 7 & 10,0 & 1,8 \\
\hline Mondeville (MON) & 5,3 & 3 & 4,3 & 1,3 \\
\hline Ifs (IFS) & 4,6 & 3 & 4,3 & 1,3 \\
\hline Colombelles (COL) & 3,1 & 3 & 4,3 & 1,3 \\
\hline Giberville (GIB) & 2,3 & 3 & 4,3 & 1,3 \\
\hline Cormelles (COR) & 2,3 & 3 & 2,9 & 0,9 \\
\hline Fleury (FLE) & 2,1 & 2 & 2,9 & 0,9 \\
\hline Bretteville (BRE) & 2,0 & 2 & 2,9 & 0,9 \\
\hline Démouville (DEM) & 1,5 & 2 & 2,9 & 0,9 \\
\hline Saint-Germain (SGE) & 1,3 & 2 & 2,9 & 0,9 \\
\hline Saint-Contest (SCO) & 1,0 & 2 & 2,9 & 0,9 \\
\hline Epron (EPR) & 0,9 & 2 & 2,9 & 0,9 \\
\hline Carpiquet (CAR) & 0,9 & 2 & 2,9 & 0,9 \\
\hline Cuverville (CUV) & 0,9 & 2 & 2,9 & 0,9 \\
\hline Louvigny (LOU) & 0,9 & 2 & 2,9 & 0,9 \\
\hline Cambes (CAM) & 0,8 & 2 & 100 & 100 \\
\hline Authie (AUT) & 0,5 & 70 & 2,9 & \\
\hline Total & 100 & & & \\
\hline
\end{tabular}

Dans le tableau 4, le résultat très important en terme d'indices de pouvoir pour Caen provient du fait que pour obtenir la majorité simple (36 voix), Caen n'a besoin de s'associer au minimum qu'à deux communes (Hérouville et une des cinq communes ayant trois délégués) et au maximum à cinq communes (ayant chacune deux délégués). Il apparaît cependant que toutes les configurations de vote ne sont pas équiprobables. L'étude historique des configurations de vote et une rencontre avec Monsieur LUC DUNCOMBE, Président de la communauté d'Agglomération du Grand Caen (anciennement district du Grand Caen), nous a permis de recenser les coalitions les plus fréquentes qui se nouent au sein de la Communauté : la coalition DURON (du nom de l'ancien député et maire de Louvigny), qui peut être présentée comme une coalition d'opposition, de gauche, sachant que la majorité des délégués de la communauté d'agglomération sont issus de partis de droite. Elle regroupe 7 communes (Cambes, Epron, Fleury, Ifs, Louvigny, Saint-Contest et Saint-Germain). La coalition du SIVOM des 3 Vallées est également une coalition de gauche, mais elle s'est surtout formée voilà plusieurs années dans l'objectif de défendre tout particulièrement l'intérêt des 5 communes la composant, ces communes formant un même "bloc" géographique contigu (partageant, entre autres, une station d'épuration). Elle regroupe les communes suivantes: Mondeville, Colombelles, Giberville, Démouville et Cuverville. La coalition Caen-Mondeville a pour sa part un caractère plus historique, basé sur l'alliance des deux maires les plus influents de la communauté d'agglomération (à l'époque Messieurs JEANMARIE GIRAULT pour la ville de Caen et JEAN-MICHEL GASNIER pour la ville de Mondeville), et ce depuis la création du district. Le cas de Mondeville s'avère être très intéressant 
à étudier. Du fait de sa forte propension à faire partie d'une coalition (elle est une commune à charges de centralité, elle est aussi très riche en taxe professionnelle et a un maire ayant un long passé politique qui l'a amené à créer la coalition Sivom), cette commune est très courtisée, et ce malgré le fait qu'elle n'ait que 3 délégués (comme Colombelles, Cormelles, Giberville et Ifs). La réalité politique actuelle fait que Mondeville forme le plus souvent la coalition du Sivom des 3 Vallées (avec Colombelles, Cuverville, Démouville et Giberville).

Tableau 5

\section{Le pouvoir de vote au sein de la Communauté d'Agglomération du Grand Caen en présence de coalitions simples}

\begin{tabular}{|c|c|c|c|}
\hline Coalition Duron & Communes & Délégués & BAN \\
\hline & CAE & 26 & 50,3 \\
\hline CAM+EPR+FLE+IFS+LOU+SCO+SGE & COAL DURON & 15 & 9,1 \\
\hline & HER & 7 & 8,7 \\
\hline & COL, COR, GIB, MON & 3 & 4,4 \\
\hline & Les autres & 2 & 2,9 \\
\hline Coalition Caen-Mondeville & Communes & Délégués & BAN \\
\hline CAE + MON & COAL CAE-MON & 29 & 93,5 \\
\hline & HER & 7 & 0,6 \\
\hline & IFS, COL, COR, GIB & 3 & 0,5 \\
\hline Coalition du SIVOM des 3 vallées & Les autres & 2 & 0,4 \\
\hline & Communes & Délégués & BAN \\
\hline MON + COL + GIB + DEM + CUV & CAE & 26 & 55,8 \\
\hline & COAL SIVOM & 13 & 6,8 \\
\hline & HER & 7 & 6,7 \\
\hline & COR, IFS & 3 & 3,8 \\
\hline
\end{tabular}

La coalition DURON affaiblit efficacement Caen mais renforce Hérouville (tableau 5). La simple alliance de Caen avec une commune ayant 3 délégués (Mondeville en l'occurrence) permet à cette coalition de réunir un important pouvoir. La coalition du SIVOM n'a d'intérêt que pour les autres communes (qui montent toutes en pouvoir), à l'exception de Caen qui baisse de manière significative.

Tableau 6

Le pouvoir de vote au sein de la Communauté d'Agglomération du Grand Caen en présence de coalitions doubles

\begin{tabular}{|c|c|c|c|}
\hline Coal. DURON/Coal. SIVOM & Communes & Délégués & BAN \\
\hline & CAE & 26 & 44,2 \\
\hline CAM+EPR+FLE+IFS+LOU+SCO+SGE & COAL DURON & 15 & 17,3 \\
\hline MON + COL + GIB + DEM + CUV & COAL SIVOM & 13 & 17,3 \\
\hline & HER & 7 & 12,5 \\
\hline & COR & 3 & 2,9 \\
\hline & AUT, BRE, CAR & 2 & 1,9 \\
\hline Coal. Caen-Mondeville/Coal. Duron & Communes & Délégués & BAN \\
\hline
\end{tabular}




\begin{tabular}{|c|c|c|c|}
\hline CAE + MON & COAL CAE-MON & 29 & 65,1 \\
\hline CAM+EPR+FLE+IFS+LOU+SCO+SGE & COAL DURON & 15 & 5,6 \\
\hline & HER & 7 & 5,6 \\
\hline & COL, COR, GIB & 3 & 3,7 \\
\hline & Les autres & 2 & 2,6 \\
\hline
\end{tabular}

La coalition DURON voit son pouvoir rehaussé, tout comme celui de la coalition SIVOM, lorsque ces deux coalitions se forment face à Caen (tableau 6). En effet la réduction du nombre des blocs de communes a un impact important sur le calcul des indices. Dans ce cas, c'est la configuration où Caen obtient le moins de pouvoir (44,2\%). Hérouville voit par contre son pouvoir augmenter de manière importante. La coalition Caen-Mondeville est efficacement affaiblie par la coalition DURON. A noter que les 7 communes de cette dernière ont, avec 15 délégués, le même pouvoir que Hérouville seule avec ses 7 délégués. En fin de compte, Hérouville a un pouvoir d'arbitrage important par rapport à la formation des coalitions DURON et SIVOM.

En conclusion, Caen pouvait afficher une grande sérénité puisqu'aucune des coalitions constatées dans la réalité politique de la communauté d'agglomération d'avant l'extension ne pouvait faire baisser de manière vraiment significative le pouvoir de la seule ville de Caen. A cela une raison très simple : il faut réunir dans une même coalition les 9 communes les plus peuplées après Caen pour pouvoir dépasser le nombre de délégués que la commune de Caen possède à elle seule (on aurait alors 26 délégués pour Caen contre 28 pour la grande coalition). Notons également que la moindre coalition que Caen accepte de former procure immédiatement à cette coalition un important pouvoir ${ }^{3}$.

Hors les problèmes de coalitions, la représentativité réelle reste le principal problème, ce qui nous a conduit à inverser notre problématique : plutôt que de déduire les indices de pouvoir de la distribution observée des sièges, pourquoi ne pas rechercher la répartition des sièges conduisant autant que faire se peut à la distribution du pouvoir souhaitée par les communes ?

\section{3) Vers la détermination d'un nombre optimal de délégués}

On se propose, à l'aide de la notion d'indice de pouvoir issue de la théorie des jeux coopératifs, d'éclairer la question du choix du nombre de délégués à affecter à chaque commune dans les structures intercommunales. Pour les structures constituées d'un petit nombre de communes, les différentes configurations possibles du pouvoir de vote sont en nombre limité et il est possible d'associer à chacune de ces configurations la ou les distributions des délégués qui lui correspondent (BISSON, BONNET, LEPELLEY, 2002). Lorsque le nombre de communes s'élève, une telle approche n'est plus possible (voir paragraphe 3.1) Nous présentons alors un algorithme (paragraphe 3.2) permettant de déterminer une distribution des délégués conduisant à une répartition du pouvoir aussi proche que possible de celle que peuvent souhaiter les élus dans l'établissement public de coopération intercommunale.

\section{1) Le dénombrement exhaustif}

Lorsque le nombre de communes s'élève à 3,4 ou 5 , il est possible de déterminer grâce à un raisonnement algébrique la liste des différentes configurations possibles du pouvoir. Par exemple pour 3 communes, il n'y a que deux configurations possibles $(1 / 3,1 / 3,1 / 3)$ et $(3 / 5,1 / 5,1 / 5)^{4}$. La structure intercommunale peut alors choisir en tout état de cause la répartition du pouvoir qui lui convient le mieux. 
A partir de 6 communes, le nombre des différentes configurations de vote est important et rend l'étude algébrique impossible. Seul l'outil informatique permet de recenser, par le calcul des indices de pouvoir associés aux différentes distributions du nombre des délégués, les différentes configurations possibles du pouvoir de vote. Pour obtenir un recensement exhaustif de ces différentes configurations il faut prendre un nombre total de délégués pair (cf. infra) et suffisamment important.

Ainsi dans le cadre d'une communauté rassemblant 6 communes, pour 24 délégués, les distributions différentes du nombre des délégués sont au nombre de 170 et elles correspondent à 80 configurations différentes du pouvoir de vote ; pour 60 délégués, les distributions différentes du nombre des délégués sont au nombre de 10467 et elles correspondent à 115 configurations différentes du pouvoir de vote ${ }^{5}$. Le tableau 7 présente le nombre de configurations différentes des indices de pouvoir selon la taille de la communauté.

Tableau 7

\section{Nombre de configurations différentes du pouvoir de vote selon la taille de la communauté}

\begin{tabular}{|c|c|c|}
\hline Nombre de communes & Parité du nombre de délégués & Nombre de configurations différentes \\
\hline \multirow{2}{*}{3} & P & 2 \\
& I & 1 \\
\hline \multirow{2}{*}{4} & P & 6 \\
& I & 2 \\
\hline \multirow{2}{*}{5} & P & 20 \\
& I & 6 \\
\hline \multirow{2}{*}{6} & P & 115 \\
& I & 20 \\
\hline \multirow{2}{*}{7} & P & 1437 \\
& I & 134 \\
\hline \multirow{2}{*}{8} & P & 12795 \\
& I & 2163 \\
\hline
\end{tabular}

Le nombre de configurations différentes du pouvoir de vote dépend de la taille de la communauté (nombre de communes la composant). Si celle-ci augmente, les configurations différentes du pouvoir de vote augmentent de manière exponentielle ${ }^{6}$. La parité du nombre total $n$ de délégués de la communauté permet d'obtenir un plus grand nombre de configurations différentes de pouvoir de vote. En effet il apparaît que pour un nombre total de délégués impair les différentes configurations de vote obtenues sont incluses dans celles obtenues avec un nombre total de délégués pair. Par exemple pour 3 communes un nombre total de délégués impair ne permet d'obtenir que la structure du pouvoir $(1 / 3,1 / 3,1 / 3)$ alors qu'avec un nombre total de délégués pair nous pouvons obtenir $(3 / 5,1 / 5,1 / 5)$ si le nombre de délégués de la plus grande commune est égal à $n / 2$ ou $(1 / 3,1 / 3,1 / 3)$ si ce dernier nombre est strictement inférieur à $n / 2$.

Cette recherche qui nécessite un dénombrement complet de toutes les distributions possibles du nombre des délégués et une comparaison des structures d'indices de pouvoir en résultant devient vite impossible en terme de temps de calcul (et ce surtout en raison de la comparaison des différentes structures d'indices de pouvoir entre elles -en l'état actuel nous l'avons limitée à 8 communes-). 
Cependant si on ne cherche pas à distinguer la répétition des différentes structures de pouvoir, le simple dénombrement de toutes les distributions du nombre des délégués (avec le calcul de l'indice de BANZHAF associé) nous permet d'aller jusqu'à 13 communes. Il est alors possible si on se fixe une cible en terme de pouvoir de vote de proposer la ou les structures de pouvoir (et la ou les distributions des délégués associées) qui rendent minimum un indice d'écart par rapport à la cible (nous retenons comme indice d'écart, la somme des valeurs absolues des écarts entre les indices de pouvoir de chaque commune et leurs cibles). Par exemple dans une communauté de communes de 13 communes si on définit la cible suivante (qui peut représenter la proportion de la population totale de chaque commune) $\{20 \%, 15 \%, 10 \%, 10 \%, 10 \%, 5 \%, 5 \%$, $5 \%, 5 \%, 5 \%, 5 \%, 4 \%, 1 \%\}$, on peut en faisant varier le nombre total de délégués, réduire l'écart à la cible pour obtenir à partir de 60 délégués la ou les distributions qui minimisent l'écart.

\section{Tableau 8}

\section{Variation de l'écart à la cible en fonction du nombre des délégués}

\begin{tabular}{|c|c|}
\hline Nombre total de délégués & Ecart à la cible \\
\hline 24 & 20,57 \\
\hline 25 & 18,62 \\
\hline 26 & 17,6 \\
\hline 27 & 15,58 \\
\hline 28 & 16,07 \\
\hline 29 & 16,21 \\
\hline 30 & 16,27 \\
\hline 31 & 16,32 \\
\hline 32 & 15,04 \\
\hline 33 & 12,96 \\
\hline 34 & 12,16 \\
\hline 35 & 12,39 \\
\hline 36 & 12,16 \\
\hline$\ldots \ldots$ & $\ldots \ldots$. \\
\hline 54 & 7,11 \\
\hline$\ldots \ldots$ & $\ldots \ldots$. \\
\hline 60 & 5,05 \\
\hline & \\
\hline
\end{tabular}

L'écart à la cible diminue en tendance lorsque le nombre des délégués augmente. En effet l'augmentation du nombre des délégués permet de proposer un plus grand nombre de distributions des délégués ce qui permet d'atteindre un plus grand nombre de structures d'indices de pouvoir parmi le nombre fini existant qui dépend uniquement de la taille (nombre de communes) de la communauté. A partir d'un nombre élevé de délégués (60) on a supposé que toutes les configurations possibles du pouvoir de vote étaient listées ${ }^{7}$. On obtient donc la ou les distributions de délégués optimale( $s$ ) car elle(s) minimise(nt) l'écart à la cible.

\section{Tableau 9}




\section{Distributions optimales du nombre des délégués}

\begin{tabular}{c|c|c|c|c|}
\cline { 2 - 5 } & Nombre de délégués & Nombre de délégués & Indices de pouvoir & Cible \\
\cline { 2 - 5 } n1 & 10 & 11 & 18,88 & 20 \\
\cline { 2 - 5 } n3 & 9 & 9 & 15,44 & 15 \\
\cline { 2 - 5 } n4 & 6 & 6 & 9,89 & 10 \\
\cline { 2 - 5 } n5 & 6 & 6 & 9,89 & 10 \\
n6 & 6 & 3 & 4,82 & 5 \\
n7 & 3 & 3 & 4,82 & 5 \\
n8 & 3 & 3 & 4,82 & 5 \\
n9 & 3 & 3 & 4,82 & 5 \\
n10 & 3 & 3 & 4,82 & 4 \\
n11 & 3 & 3 & 4,82 & 1 \\
n12 & 3 & 1 & 2,27 & 5,05 \\
n13 & 3 & 60 délégués & Ecart & 5 \\
\hline Total & 60 délégués & & &
\end{tabular}

Dans l'exemple précédent (tableau 9) il y a 2 distributions différentes du nombre des délégués qui donnent la même structure d'indices de pouvoir, cette dernière minimisant l'écart à la cible qui est alors de 5,05. Afin d'éviter le recensement exhaustif de toutes les distributions différentes des délégués et de leurs indices de pouvoir associés, la mise au point d'un algorithme va nous permettre de converger dans la quasi-totalité des cas vers la solution optimale ou à défaut vers une solution acceptable.

\subsection{L’algorithme dans le cas général}

L'algorithme présenté dans ce paragraphe s'inspire de ceux de LEECH (2001) et LARUELLE (1998). Il s'en différencie cependant par la prise en compte d'une contrainte supplémentaire liée au caractère entier des poids associés aux différents joueurs : en effet, dans le contexte étudié, les poids des joueurs (les communes) correspondent aux nombres de leurs délégués -entiers par définition-.

On suppose que la communauté se fixe un vecteur cible constitué des indices de pouvoir souhaités pour chacune des communes. Soi $T_{i} l^{\prime}$ indice de pouvoir souhaité pour la commune $i$; on note $\beta_{i}$ l'indice de pouvoir de la commune $i$ obtenu en appliquant l'indice de Banzhaf à une répartition quelconque des délégués. L'objectif est d'obtenir la répartition des délégués permettant de rapprocher autant que faire se peut les indices obtenus ( $\left.\beta_{i}\right)$ des indices désirés $\left(T_{i}\right)$. Pour y parvenir, on pose $S_{i}=\left|T_{i}-\beta_{i}\right|$, ce qui correspond pour toute commune $i$ à la différence en valeur absolue entre l'indice souhaité et l'indice obtenu. Plus $\mathrm{S}_{i}$ est élevé, moins l'indice obtenu par la commune $i$ est conforme à ce qui était attendu (en sa faveur comme en sa défaveur). L'objectif final est de minimiser la somme des $\mathrm{S}_{\mathrm{i}}$. On procède par itération de la manière suivante :

-on choisit un point de départ (une répartition initiale des délégués) -le choix du point de départ est discuté en annexe 1-, 
-on calcule la somme des écarts à la cible $\sum S_{i}$,

-on fait varier les nombres de délégués de chaque commune par couple en retirant un délégué à une commune et en ajoutant un délégué à une autre commune (les autres communes gardent le même nombre de délégués $)^{8}$. Pour chaque couple on calcule la somme $\sum S_{i}$ associée et on retient la répartition des délégués associée à la somme la plus faible.

-on réitère le processus jusqu'à ce qu'il ne soit plus possible de réduire la somme $\sum S_{i}$. Dans ce cas nous avons obtenu la répartition que nous considérons comme optimale 9 .

\section{Application aux structures intercommunales bas-normandes}

Dans le cas des communautés de communes et des communautés d'agglomération la répartition du nombre de sièges par commune se fait à l'amiable, la loi préconisant de prendre la population comme indicateur de référence. Le conseil communautaire est alors constitué par un certain nombre de délégués élus au sein de chaque conseil municipal pour représenter leur commune. Les maires ont toutefois tout pouvoir de proposer (ou non) une représentation des listes minoritaires au sein de la délégation municipale qui va siéger au conseil communautaire. Il existe deux types de décision au sein des communautés de communes :

-les décisions prises à la majorité simple et qui concernent la gestion courante de la structure intercommunale. Le calcul des indices de pouvoir est immédiat et il permet de mesurer le pouvoir de décision d'une commune en supposant que les délégués de la commune votent toujours en bloc,

-les décisions prises à la majorité qualifiée qui sont requises lorsqu'une décision modifie les statuts communautaires (modification du périmètre communautaire, extension du domaine des compétences communautaires dans le cas des communautés de communes etc.). Cette majorité qualifiée est un peu plus complexe car une proposition est acceptée si elle réunit la moitié des communes (vote de la proposition au sein de chaque conseil municipal) représentant les $2 / 3$ de la population de 1'E.P.C.I. ou les $2 / 3$ des communes représentant la moitié de la population de l'E.P.C.I.. On peut cependant montrer que cette décision revient à construire les indices de pouvoir en deux étapes. La première étape considère que chaque conseil municipal peut se prononcer pour ou contre une décision ce qui revient à donner à chaque commune une pondération unitaire. La seconde étape consiste à vérifier que les coalitions respectant le nombre de communes vérifient aussi les quotas de population requis.

Dans le cas des communautés d'agglomération, la définition et l'extension des compétences communautaires est prise au sein du conseil communautaire à la majorité des 2/3.

\subsection{Exemple d'une communauté de communes : la CDC de Lessay}

Majorité qualifiée Nous n'avons bien évidemment pas exploré le vote au sein de chaque conseil municipal mais nous pouvons former les coalitions gagnantes qui respectent les conditions requises ( $1 / 2$ et $2 / 3$ ou $2 / 3$ et $1 / 2$ avec en plus dans le cas des communautés de communes toute commune représentant plus de $25 \%$ de la population totale -ce qui lui donne de fait un droit de veto-). Dans ces coalitions gagnantes nous regardons les communes qui sont critiques (en terme de population mais aussi en terme de nombre de communes). Dans ce dernier cas soit toutes les communes de la coalition sont critiques, soit aucune ne l'est du fait de leur statut identique (tableau 10). 
Tableau 10

Calcul des indices de pouvoir dans le cas de la majorité qualifiée pour la communauté de communes de Lessay

\begin{tabular}{|c|c|c|c|}
\hline Nom commune & $\begin{array}{c}\text { Population } \\
\text { Insee }\end{array}$ & $\begin{array}{c}\text { Pourcentage } \\
\text { Population }\end{array}$ & $\begin{array}{c}\text { Indices de pouvoir à la } \\
\text { majorité qualifiée }\end{array}$ \\
\hline Créances & 2014 & 22,71 & 15,83 \\
\hline Lessay & 1763 & 19,88 & 14,96 \\
\hline Pirou & 1313 & 14,80 & 10,14 \\
\hline St Germain/Ay & 797 & 8,99 & 8,15 \\
\hline Millières & 562 & 6,34 & 6,94 \\
\hline Vesly & 541 & 6,10 & 6,89 \\
\hline Bretteville/Ay & 399 & 4,50 & 6,07 \\
\hline Geffosses & 324 & 3,65 & 5,60 \\
\hline Anneville & 317 & 3,57 & 5,60 \\
\hline La Feuillue & 263 & 2,97 & 5,19 \\
\hline Angoville/Ay & 230 & 2,59 & 5,14 \\
\hline Laulne & 183 & 2,06 & 4,75 \\
\hline St Patrice de Clauls & 163 & 1,84 & 4,73 \\
\hline Total & 8869 & 100 & 100 \\
\hline
\end{tabular}

La majorité qualifiée permet de distinguer la représentativité de chaque commune grâce à la prise en compte de sa population. On obtient des indices de pouvoir qui reflètent globalement la hiérarchie de la représentativité en terme de poids de chaque commune dans la population totale. On notera cependant dans le cas de la CDC de Lessay que les communes les plus peuplées sont un peu sous-représentées (contrainte du nombre de communes) tandis que les communes les moins peuplées sont plutôt sur-représentées.

Majorité simple En ce qui concerne les décisions courantes de gestion au sein de l'E.P.C.I., les décisions se font à la majorité simple au sein du conseil communautaire (tableau 11). Nous suivons donc la procédure présentée dans le calcul de l'indice de BANZHAF afin de recenser les coalitions gagnantes en fonction du nombre de délégués qu'a obtenus chaque commune au sein du conseil communautaire. 
Tableau 11

Calcul des indices de pouvoir dans le cas de la majorité simple pour la communauté de communes de Lessay

\begin{tabular}{|c|c|c|c|}
\hline Nom commune & $\begin{array}{c}\text { Nombre de } \\
\text { délégués }\end{array}$ & $\begin{array}{c}\text { Indices de } \\
\text { pouvoir }\end{array}$ & $\begin{array}{c}\text { Pourcentage } \\
\text { population }\end{array}$ \\
\hline Créances & 6 & 17,07 & 22,71 \\
\hline Lessay & 5 & 13,09 & 19,88 \\
\hline Pirou & 5 & 13,09 & 14,80 \\
\hline St Germain/Ay & 3 & 7,57 & 8,99 \\
\hline Millières & 3 & 7,57 & 6,34 \\
\hline Vesly & 3 & 7,57 & 6,10 \\
\hline Bretteville/Ay & 2 & 4,86 & 4,50 \\
\hline Geffosses & 2 & 4,86 & 3,65 \\
\hline Anneville & 2 & 4,86 & 3,57 \\
\hline La Feuillue & 2 & 4,86 & 2,97 \\
\hline Angoville/Ay & 2 & 4,86 & 2,59 \\
\hline Laulne & 2 & 4,86 & 2,06 \\
\hline St Patrice de Clauls & 2 & 4,86 & 1,84 \\
\hline Total & 39 & 100 & 100 \\
\hline
\end{tabular}

Dans la répartition actuelle du nombre de délégués de la communauté, on peut constater que la différenciation effectuée se retrouve bien en terme d'indices de pouvoir puisque quel que soit le nombre de délégués $(2,3,5$ ou 6$)$ les indices de pouvoir sont différents. Créances avec 6 délégués obtient $17,07 \%$ du pouvoir de vote à la majorité simple et ce devant Pirou et Lessay qui ont chacune 5 délégués et $13,09 \%$ de pouvoir de vote.

Nous avons aussi calculé une distribution optimale du nombre des délégués par commune dans le cadre de la majorité simple (tableau 12) si on considère que l'indice de pouvoir d'une commune doit être en adéquation parfaite avec la population. Elle consiste à minimiser la somme des valeurs absolues des écarts de la population de chaque commune (en pourcentage) et de l'indice de pouvoir associé (colonne Indices de pouvoir 1).

Tableau 12

Distribution optimale du nombre de délégués dans le cas de la majorité simple pour la communauté de communes de Lessay

\begin{tabular}{|c|c|c|c|c|c|}
\hline Nom commune & $\begin{array}{c}\text { Optimum } \\
\text { délégués }\end{array}$ & $\begin{array}{c}\text { Indices de } \\
\text { pouvoir 1 }\end{array}$ & $\begin{array}{c}\text { Optimum } \\
\text { délégués avec 1 }\end{array}$ & $\begin{array}{c}\text { Indices de } \\
\text { pouvoir 2 }\end{array}$ & $\begin{array}{c}\text { Pourcentage } \\
\text { population }\end{array}$ \\
\hline Créances & 7 & 20,53 & 8 & 22,23 & 22,71 \\
\hline Lessay & 7 & 20,53 & 7 & 18,51 & 19,88 \\
\hline Pirou & 5 & 15,43 & 6 & 15,71 & 14,80 \\
\hline St Germain/Ay & 2 & 4,35 & 4 & 9,49 & 8,99 \\
\hline Millières & 2 & 4,35 & 3 & 7,34 & 6,34 \\
\hline Vesly & 2 & 4,35 & 3 & 7,34 & 6,10 \\
\hline Bretteville/Ay & 2 & 4,35 & 2 & 4,80 & 4,50 \\
\hline Geffosses & 2 & 4,35 & 1 & 2,43 & 3,65 \\
\hline Anneville & 2 & 4,35 & 1 & 2,43 & 3,57 \\
\hline La Feuillue & 2 & 4,35 & 1 & 2,43 & 2,97 \\
\hline Angoville/Ay & 2 & 4,35 & 1 & 2,43 & 2,59 \\
\hline Laulne & 2 & 4,35 & 1 & 2,43 & 2,06 \\
\hline
\end{tabular}




\begin{tabular}{|c|c|c|c|c|c|}
\hline St Patrice de Clauls & 2 & 4,35 & 1 & 2,43 & 1,84 \\
\hline Total & 39 & 100 & 39 & 100 & 100 \\
\hline
\end{tabular}

Ainsi pour cette communauté la situation actuelle donne un indicateur de 31,12 et l'optimum obtenu permet de réduire cette valeur à 21,40 si on garde le même nombre de délégués (39). Si la communauté accepte une représentation de certaines communes avec un seul délégué cet écart peut être nettement réduit puisque l'on obtient la valeur de 9,80 (ce qui correspond à la somme des valeurs absolues des écarts entre la colonne Pourcentage population et la colonne Indices de pouvoir 2). Bien entendu d'autres considérations peuvent entrer en ligne de compte dans la répartition du nombre des délégués. Si toutefois on voulait encore réduire cet écart, il faudrait effectuer des recherches sur un nombre total de délégués différent (soit plus, soit moins de 39 délégués) et en particulier avec un nombre total de délégués pair. Le nombre des répartitions différentes de pouvoir de vote étant dans ce cas plus important, il est probable que l'on puisse encore réduire l'indicateur d'écart à la cible.

\subsection{Exemple d'une communauté d'agglomération : la CA du Pays de Flers}

Dans le cas des communautés d'agglomérations et des communautés urbaines, l'extension du domaine des compétences se fait à la majorité des $2 / 3$ des délégués au sein du conseil communautaire. Pour les communautés d'agglomérations ou urbaines la commune représentant plus de la moitié de la population doit aussi faire partie de la coalition ou à défaut la commune la plus peuplée.

Majorité qualifiée. La majorité qualifiée permet théoriquement de distinguer la représentativité de chaque commune grâce à la prise en compte de sa population. On obtient cependant des indices de pouvoir identiques pour toutes les communes autres que la communecentre de Flers (tableau 13).

Tableau 13

Calcul des indices de pouvoir dans le cas de la majorité qualifiée et de la majorité aux 2/3 pour la communauté d'agglomération du Pays de Flers

\begin{tabular}{|c|c|c|c|c|c|}
\hline Nom commune & $\begin{array}{c}\text { Population } \\
\text { Insee }\end{array}$ & $\begin{array}{c}\text { Pourcentage } \\
\text { Population }\end{array}$ & $\begin{array}{c}\text { Indices de pouvoir à } \\
\text { la majorité qualifiée }\end{array}$ & $\begin{array}{c}\text { Nombre de } \\
\text { délégués }\end{array}$ & $\begin{array}{c}\text { Majorité } \\
\text { aux 2/3 }\end{array}$ \\
\hline Flers & 16947 & 59,49 & 31,16 & 15 & 39,39 \\
\hline St Georges des Groseillers & 3259 & 11,44 & 5,74 & 4 & 14,87 \\
\hline La Lande-Patry & 1757 & 6,17 & 5,74 & 3 & 10,26 \\
\hline La Selle La Forge & 1277 & 4,48 & 5,74 & 2 & 6,42 \\
\hline Caligny & 870 & 3,05 & 5,74 & 1 & 3,23 \\
\hline St Clair de Halouze & 845 & 2,97 & 5,74 & 1 & 3,23 \\
\hline Montilly/Noireau & 763 & 2,68 & 5,74 & 1 & 3,23 \\
\hline Cerisy Belle Etoile & 688 & 2,42 & 5,74 & 1 & 3,23 \\
\hline Chapelle-au-Moine & 633 & 2,22 & 5,74 & 1 & 3,23 \\
\hline Chapelle-Biche & 480 & 1,69 & 5,74 & 1 & 3,23 \\
\hline Landigou & 439 & 1,54 & 5,74 & 1 & 3,23 \\
\hline Aubusson & 335 & 1,18 & 5,74 & 1 & 3,23 \\
\hline La Bazoque & 192 & 0,67 & 5,74 & 1 & 3,23 \\
\hline Total & 28485 & 100 & 100 & 33 & 100 \\
\hline
\end{tabular}


La différence importante entre la population de cette commune et les populations des autres communes explique cette non-différenciation en terme d'indices de pouvoir. En effet toutes les communes autres que la commune-centre ne peuvent réunir la moitié de la population de la communauté d'agglomération et a fortiori les $2 / 3$ ce qui explique leur statut identique. Il reste que la contrainte du nombre de communes explique la sous-représentation du pouvoir de vote de Flers si on la compare à son poids démographique.

A la majorité aux $2 / 3$ c'est le nombre de délégués de chaque commune au sein du conseil communautaire qui intervient dans la détermination des indices de pouvoir. Nous suivons donc la procédure présentée dans l'exemple précédent de Lessay afin de recenser les coalitions gagnantes en fonction du nombre de délégués qu'a obtenu chaque commune au sein du conseil communautaire en prenant toutefois pour seuil $2 / 3$ et non $1 / 2$. On peut constater que la différenciation effectuée se retrouve bien en terme d'indices de pouvoir puisque quel que soit le nombre de délégués $(1,2,3,4$ ou 15), les indices de pouvoir sont différents. Flers avec 15 délégués obtient $39,39 \%$ du pouvoir de vote devant Saint-Georges-des-Groseillers avec $14,87 \% \ldots$

Majorité simple. En ce qui concerne les décisions courantes de gestion au sein de l'EPCI, elles se prennent à la majorité simple au sein du conseil communautaire (tableau 14).

\section{Tableau 14}

\section{Calcul des indices de pouvoir dans le cas de la majorité simple pour la communauté d'agglomération du Pays de Flers}

\begin{tabular}{|c|c|c|c|c|}
\hline Nom commune & $\begin{array}{c}\text { Population } \\
\text { Insee }\end{array}$ & $\begin{array}{c}\text { Pourcentage } \\
\text { Population }\end{array}$ & $\begin{array}{c}\text { Nombre de } \\
\text { délégués }\end{array}$ & $\begin{array}{c}\text { Indices de } \\
\text { pouvoir }\end{array}$ \\
\hline Flers & 16947 & 59.49 & 15 & 95.23 \\
\hline St Georges des Groseillers & 3259 & 11.44 & 4 & 0.47 \\
\hline La Lande-Patry & 1757 & 6.17 & 3 & 0.47 \\
\hline La Selle La Forge & 1277 & 4.48 & 2 & 0.47 \\
\hline Caligny & 870 & 3.05 & 1 & 0.37 \\
\hline St Clair de Halouze & 845 & 2.97 & 1 & 0.37 \\
\hline Montilly/Noireau & 763 & 2.68 & 1 & 0.37 \\
\hline Cerisy Belle Etoile & 688 & 2.42 & 1 & 0.37 \\
\hline Chapelle-au-Moine & 633 & 2.22 & 1 & 0.37 \\
\hline Chapelle-Biche & 480 & 1.69 & 1 & 0.37 \\
\hline Landigou & 439 & 1.54 & 1 & 0.37 \\
\hline Aubusson & 335 & 1.18 & 1 & 0.37 \\
\hline La Bazoque & 192 & 0.67 & 33 & 100 \\
\hline Total & 28485 & 100 & 1 & 0.37 \\
\hline
\end{tabular}

Dans la répartition actuelle du nombre de délégués de la communauté, on peut constater que la différenciation effectuée ne se retrouve qu'imparfaitement en terme d'indices de pouvoir. En effet Saint-Georges-des-Groseillers, La Lande-Patry et La Selle-La-Forge, avec respectivement 4, 3 et 2 délégués, obtiennent chacune le même indice de pouvoir, à savoir 0,47\% du pouvoir de vote tandis que Flers obtient la quasi-totalité du pouvoir de vote car il lui suffit d'attirer une commune au minimum et deux au maximum pour emporter la décision. 
Si on considère que l'indice de pouvoir d'une commune doit être en adéquation parfaite avec sa population, la distribution optimale trouvée permet de minimiser l'indicateur d'écart par rapport à la cible (tableau 15).

\section{Tableau 15 \\ Distribution optimale du nombre de délégués dans le cas de la majorité simple pour la communauté d'agglomération du Pays de Flers}

\begin{tabular}{|c|c|c|c|c|}
\hline Nom commune & $\begin{array}{c}\text { Population } \\
\text { Insee }\end{array}$ & $\begin{array}{c}\text { Pourcentage } \\
\text { Population }\end{array}$ & $\begin{array}{c}\text { Optimum avec } \\
1\end{array}$ & $\begin{array}{c}\text { Indices de } \\
\text { pouvoir }\end{array}$ \\
\hline Flers & 16947 & 59.49 & 12 & 59,38 \\
\hline St Georges des Groseillers & 3259 & 11.44 & 5 & 6,32 \\
\hline La Lande-Patry & 1757 & 6.17 & 3 & 5,81 \\
\hline La Selle Le Forge & 1277 & 4.48 & 2 & 4,33 \\
\hline Caligny & 870 & 3.05 & 2 & 4,33 \\
\hline St Clair de Halouze & 845 & 2.97 & 2 & 4,33 \\
\hline Montilly/Noireau & 763 & 2.68 & 1 & 2,21 \\
\hline Cerisy Belle Etoile & 688 & 2.42 & 1 & 2,21 \\
\hline Chapelle-au-Moine & 633 & 2.22 & 1 & 2,21 \\
\hline Chapelle-Biche & 480 & 1.69 & 1 & 2,21 \\
\hline Landigou & 439 & 1.54 & 1 & 2,21 \\
\hline Aubusson & 335 & 1.18 & 1 & 2,21 \\
\hline La Bazoque & 192 & 0.67 & 1 & 2,21 \\
\hline Total & 28485 & 100 & 33 & 100 \\
\hline
\end{tabular}

Ainsi, pour cette communauté, la situation actuelle donne un indicateur de 71,48 et l'optimum obtenu permet de réduire cette valeur à 12,84. On notera cependant que si Flers obtient un indice de pouvoir très proche de son poids démographique, Saint-Georges-desGroseillers reste sous-représentée. Si on désire augmenter l'indice de pouvoir de cette dernière commune, ce sera au détriment de l'indice de pouvoir de Flers (et ce plus que proportionnellement). Il apparaît donc dans ce cas une certaine incompatibilité, c'est-à-dire qu'une augmentation du pouvoir de vote de Saint Georges des Groseillers se traduira obligatoirement par une diminution du pouvoir de vote de Flers.

\section{Conclusion}

Le calcul des indices de pouvoir ignore délibérément certains aspects psychologiques et politiques du processus démocratique et ne conduit qu'à une évaluation a priori du pouvoir. En particulier la répartition des vices-présidents et de leurs attributions constitue un élément important au même titre que les associations préférentielles entre communes. L'analyse présentée dans cet article n'éclaire donc qu'un aspect de la réalité du pouvoir de décision des communes.

Cependant, le calcul des indices de pouvoir garde tout son sens lorsque la structure intercommunale doit se créer ou s'agrandir. Il permet en effet de constituer une référence objective susceptible de servir de base à la négociation relative à l'attribution d'un nombre de délégués pour chaque commune. Ces méthodes sont d'ailleurs utilisées dans le cadre européen (Conseil des ministres et Parlement) afin de mesurer la représentation de chaque Etat. Elles ont joué un rôle important dans les négociations du traité de Nice et peut-être un rôle insuffisant lors de l'échec du récent sommet de Bruxelles. Nous pensons pour notre part que le recours aux méthodes de la théorie des jeux dans le cadre de l'analyse de la représentativité des différentes 
communes au sein des structures intercommunales mérite d'être largement développé à des fins à la fois normatives et positives.

Bibliographie

ADCF, 2001, "Les élus intercommunaux jugent l'intercommunalité", consultation nationale de l'Assemblée des Communautés de France, 182 rue de Rivoli, 75001 Paris, octobre.

ADCF, 2000, "Enquête de l'Assemblée des Communautés de France (ADCF)", Groupe Caisse d'Epargne, octobre.

ADCF, 1999, "Annuaire de l'intercommunalité 1999", de l'Assemblée des Districts et des Communautés de France (ADCF), 182 rue de Rivoli, 75001 Paris.

BANZHAF, J., 1965, "Weighted Voting Doesn't Work : A Mathematical Analysis", Rutgers Law Review, 19, 317-343.

BONNET J. et D. LEPELLEY, 2001, "Pouvoir de vote et intercommunalité: le cas des E.P.C.I. de Basse-Normandie", in Démocratie et management local, R. Le Duff, J.P. Rigal et G. Schmidt éditeurs, Dalloz.

BISSON F., BONNET J. et D. LEPELLEY, 2002, "Pouvoir et coalitions au sein des structures intercommunales: l'éclairage de la théorie des jeux coopératifs", XXXVIIIième colloque de l'ASRDLF (Association de Science Régionale de Langue Française),Trois-Rivières, Québec, 2122-23 août.

BOURDAN, P., 1995, L'intercommunalité en Basse-Normandie, rapport pour le Conseil Economique et Social Régional.

FELSENTHAL D. AND M. MACHOVER, 1998, The Measurement of Voting Power, Edward Elgar Publishing.

KHUN A., 2001, "La nouvelle figure de la gouvernance territoriale en milieu rural", in Démocratie et management local, R. Le Duff, J.P. Rigal et G. Schmidt éditeurs, Dalloz.

LARUELLE A., 1998, Game Theoretical Analysis of Decision-Making Processes with Applications to the European Union, Thèse de doctorat, Université Catholique de Louvain.

LARUELLE A. and M. WIDGRÉN, 1998, "Is the Allocation of Voting Power Among the EU States Fair", Public Choice, 94, 317-339.

LEECH D., 2001, "Designing the voting system for the council of the European Union", Department of economics and CSGR, University of Warwick, à paraître in Public Choice.

MOULIN M., 2002, L'intercommunalité en Basse-Normandie, rapport présenté au Conseil Economique et Social Régional, Décembre.

SHAPLEY L.S. and M. SHUBIK, 1954, "A Method for Evaluating the Distribution of Power in a Committee System", American Political Science Review, 48, 787-792.

VALLETOUX F., 1997, "Les élus locaux attendent une réforme de l'intercommunalité", Enquête du Forum pour la gestion des villes, les Echos du 25 septembre.

WIDGRÉN M., 1994, "Voting Power in the EC and the Consequences of Two Different Enlargements", European Economic Review, 38, 1153-1170. 
Annexe 1 : Points de départ et convergence de l'algorithme

Soit $m$ le nombre de communes de la communauté. Soit $n_{i}$ le nombre de délégués de la commune $i . n=\sum n_{\mathrm{i}}$ désigne le nombre total de délégués de la communauté. Les communes sont classées dans l'ordre décroissant de leur population.

Deux points de départ de l'algorithme sont a priori imaginables,

-Le point de départ proportionnel qui est la répartition des délégués se rapprochant le plus possible du vecteur cible.

Pour tout réel $\mathrm{x}$, on note $[\mathrm{x}]$ l'arrondi de x à l'entier le plus proche. Soit

$$
\begin{aligned}
& \alpha=\left\{\begin{array}{l}
\frac{n}{2}-1 \text { si } n \text { estpair. } \\
\frac{n-1}{2} \text { si } n \text { estimpair. }
\end{array} \quad\right. \text { On pose alors : } \\
& n_{1}=\min \quad\left[T_{1}\right] \\
& n_{m}=\max \left\{1,\left[\frac{T_{m}\left(n-n_{1}\right)}{1-T_{1}}\right]\right\} \\
& n_{i}=\max \left\{1,\left[\frac{T_{i}\left(n-n_{1}-\sum_{j=i+1}^{n} n_{j}\right)}{1-T_{i}-\sum_{j=i+1}^{m} T_{j}}\right]\right\} \text { pour } i=m-1, m-2, \ldots, 3 \text {. } \\
& n_{2}=n-n_{1-} \sum_{i=3}^{m} n_{\mathrm{i}}
\end{aligned}
$$

-Le point de départ neutre qui s'efforce d'attribuer à chaque commune le même nombre de délégués $(\mathrm{n} / \mathrm{m})$. Pour ce point de départ il appartient à l'utilisateur d'effectuer les arrondis appropriés.

La convergence dépend en partie du point de départ. Dans les applications de l'algorithme nous avons systématiquement utilisé les deux points de départ. Nous avons testé pour une communauté de 10 communes la convergence et la rapidité de l'algorithme en faisant varier le nombre de délégués de 20 à 40 et en prenant aussi un nombre total de délégués de 50 (dans le cas de 10 communes, on peut par énumération déterminer la distribution optimale et donc apprécier la qualité de notre algorithme).

Deux cibles différentes ont été choisies :

-la cible $1:\{40 \%, 17 \%, 12 \%, 10 \%, 6 \%, 5 \%, 4 \%, 3 \%, 2 \%, 1 \%\}$,

-la cible $2:\{60 \%, 11 \%, 7 \%, 5 \%, 5 \%, 4 \%, 3 \%, 2 \%, 2 \%, 1 \%\}$.

En ce qui concerne la première cible, dans 18 cas sur 22, le point de départ neutre permet d'obtenir l'optimum. En prenant comme point de départ le point proportionnel, nous avons aussi dans 18 cas sur 22 une convergence vers l'optimum. Au total, si on considère les deux points de départ, on trouve l'optimum dans 21 cas sur 22, ce qui illustre bien la complémentarité des deux points de départ. Le cas pour lequel nous n'avons pas obtenu l'optimum est le cas où le nombre de délégués est de 50. Les résultats obtenus sont cependant proches de l'optimum : si l'on classe les 16289 différentes distributions possibles des délégués par ordre croissant de distance à la 
cible, on obtient la 3ième position en prenant le point de départ neutre et la 4ième en prenant le point de départ proportionnel.

En ce qui concerne la deuxième cible, on trouve l'optimum dans tous les cas grâce notamment à la complémentarité des deux points de départ. Comme on pouvait s'y attendre, la vitesse de la convergence est plus importante pour le point de départ proportionnel (en moyenne 2,45 itérations contre 10,45 pour la première cible et 5,05 itérations contre 10,36 pour la seconde cible).

\section{RESUME:}

Cet article propose une étude de la représentativité des communes au sein des différents établissements publics de coopération intercommunale (EPCI) bas-normands. Il utilise pour cela la notion d'indice de pouvoir (au sens de Banzhaf), outil de la théorie des jeux coopératifs qui permet de mesurer le pouvoir de vote dans le cas d'une décision prise à la majorité avec quota. Après une étude de la situation bas-normande, les auteurs s'intéressent à l'existence de coalitions préférentielles (qui nuancent le calcul a priori des indices de pouvoir) et à la recherche d'une distribution optimale par commune du nombre des délégués. Cette dernière permet de corriger les écarts de représentativité souvent constatés dans la réalité bas-normande.

\section{SUMMARY:}

We investigate in this paper the representation of the cities inside the french local entities called E.P.C.I. (Etablissement Public de Cooperation Intercommunale). In these entities, each city is represented by a given number of delegates. Our analysis focuses on E.PC.I.s from BasseNormandie and is based upon the notion of voting power index (in the sense of Banzhaf), borrowed from cooperative game theory. Our calculations take into account the fact that some coalitions are more likely to occur than others. It turns out that the voting power of cities is often different from their relative size, as measured by their population. We finally propose an algorithm allowing the determination of the "optimal" number of delegates for each city.

\footnotetext{
${ }^{1}$ Les communautés urbaines se distinguent cependant avec une double représentation proportionnelle (pour le nombre de délégués de chaque commune) et au sein de chaque commune avec la prise en compte des listes minoritaires.

2 Depuis décembre 2002, la communauté s'est agrandie (elle est passée à 28 communes) et elle a changé de dénomination (elle se dénomme actuellement Caen la Mer). Il est un peu prématuré d'envisager les différentes coalitions au sein de cette nouvelle communauté.

${ }^{3}$ De fait Madame le Maire de Caen (Madame LEBRETHON), consciente du poids très important de Caen au sein de la communauté d'agglomération, a accordé deux sièges (sur les 26) aux listes minoritaires ainsi que la loi le lui permet. On pourrait alors supposer que pour certaines décisions ces deux élus se désolidarisent du vote en bloc de la commune de Caen. Nous n'avons pas exploré plus avant ce cas de figure.

${ }^{4}$ voir BISSON, BONNET, LEPELLEY, 2002. L'analyse proposée considère des votes à la majorité simple soit un quota de $50 \%$ du nombre total des délégués (plus un si ce nombre est pair).

${ }^{5}$ Nous avons vérifié que si on augmente le nombre de délégués on ne trouve pas de nouvelles configurations du pouvoir de vote.

${ }^{6}$ Pour les communautés de 6,7 ou 8 communes, les calculs ont été faits avec 60 délégués dans le cas de la parité et 59 délégués lorsque le nombre global de délégués est impair. Ces nombres de délégués devraient être suffisamment importants pour nous permettre de recenser toutes les configurations différentes du pouvoir de vote.
} 
${ }^{7}$ Ce n'est pas certain car une communauté de 13 communes doit permettre d'atteindre un nombre très élevé de structures d'indices de pouvoir différentes.

${ }^{8}$ Cette opération doit prendre en compte certaines contraintes : par exemple une commune ne peut recevoir plus de la moitié du nombre des délégués, de la même façon on ne peut accepter qu'une commune ne puisse avoir aucun délégué.

${ }^{9}$ Notons que l'algorithme ne converge pas toujours vers l'optimum optimorum. L'expérience montre cependant que, dans les cas peu fréquents où le minimum global n'est pas atteint, le minimum local proposé par l'algorithme n'en est pas éloigné (voir sur ce point l'annexe 1). 